\title{
Design and Development of Simple Guiding Blood Pressure Measurement (SGBPM) Mobile Application
}

\author{
E. Hemavathy", S. Sinthuja and N. Manoharan \\ AMET University, Chennai, India; hemavathy47@gmail.com, \\ sinthuja.engg@gmail.com, Directorresearch@ametuniv.ac.in
}

\begin{abstract}
Due to the fast growing population of aged people, remote health monitoring becomes highly essential. An increasing number of users are surrounded by multiple computing devices such as PDAs and cell phones. Use of mobile devices in health care might improve decision-making and reduce the numbers of medical errors but, it is not commonly used in telemedicine and health care, due to the lack of suitable functions and software applications. The present study is to develop a mobile guiding system with User Interface for blood pressure monitoring. On the other hand in the 3rd era of computing, wireless and remote health monitoring solution provides simple, compact, robust and cheaper solution to different categories of patient. In this project a simple and convenient blood pressure measurement for aged with efficient guiding tool design which is the mobile phone application for health management and self monitoring on the top of Java 2 Micro Edition can be achieved.
\end{abstract}

Keywords: Blood Pressure Measurement, LabVIEW, J2ME, Guiding System

\section{Introduction}

In medical, remote monitoring encompasses a number of high-tech applications that involve providing remote care for people, in terms of clinical conditions that can be monitored, how often they should be monitored and whether they should be monitored real-time or periodically. Typically at home, where he or she can connect to the wide range of home care applications accessible to the referring physician or family members who can monitor by connected to a medical device ${ }^{1}$. Remote guidance is the supervision or guidance of a medical task i.e. a procedures or test from a remote location, providing diagnose to the patient without physical present near the patient. After such monitoring, a clinical guidance on whether the patient requires immediate hospitalization, urgent doctor visit, and any control measures to maintain their health condition. Remote patient monitoring technologies are safer, more effective monitoring of health and safety among older adults, control visits to hospitals with an objective of addressing cost, decongestion issues, chronicailments, geographic challenges, geriatric conditions and lack of mobility ${ }^{2}$.

At the global level, 60-plus has risen to around 11\% (760 million) in 2011, and still increases expected to reach $22 \%$ ( 2 billion) by 2050 and there will be over 1 billion people aged 60 and older in Asia by $2050^{3}$. When these numbers are placed in the context of health services for older people, there is a growing apprehension of how current health systems infrastructure, health personnel, and budgets will meet the increasing demands for these services.

Mobile phones provide a particularly promising platform for health management applications due to their central role in people's lives as well as their technical capabilities this paper presents a mobile phone application

${ }^{*}$ Author for correspondence 
for supporting the self-management of health, targeting working-age citizens and well being.

There are many programming languages available today for developing mobile applications. Some of these technologies include Java 2 Micro Edition (J2ME), Python, $\mathrm{C} / \mathrm{C}++$, Easy Mobile Programming (EMP), etc. Of all these languages, the J2ME stands out as the technology of choice because it is platform independent and has a rich set of APIs appropriate for mobile devices. Consequently, the guiding system solution for blood pressure developed in this work made use of the Java 2 Micro Edition (J2ME) ${ }^{4}$.The increasing prevalence of lifestylerelated health risks coupled with the lack of resources in preventative healthcare calls for novel and cost-efficient methods for supporting citizens in their independent health and well-being management. The challenge is to develop efficient methods that support citizens in their health management and empower them to take more responsibility for their health.

Some of healthcare models such as Health Buddy model where patient responses are color-coded by risk level as High (red), Moderate (yellow), and Low (green). Telestation Patients model answer health assessment survey questions and information is transmitted through the phone line to health care professionals 5 .Genesis DM model is a web-based system with customizable subjective disease-related queries for patients. Intel's Health Guide model combines in-home patient device with an online interface where patients and caregivers interact using two-way audio and video ${ }^{6}$. Life View American model also combines patient monitoring and interactive video,the status of the patients is monitored by the healthcare professionals, data are collected from peripheral devices, patient monitors, then transmitted through telephone line or broadband connection, two-way audio/ video allows clinicians and patients to communicate ${ }^{7}$. Ideal LIFE Pod model data are collected from peripheraldevice, which then transmits data through an analog phone line. Health anywhere model provides questionnaires, and two-way video that link patients with healthcare professionals, data can be viewed by healthcare professionals and users. It is also integrated with Microsoft's Health Vault. Personalized comments service also proposed for hypertension patients using mobile device ${ }^{8}$. Existing systems monitor patients, updates information helpful for doctors but still patients have no awareness on control of their health condition, diet, medicine and about their dangerous zone. Hence the new system is proposed.

\section{System Architecture}

To reduce the emergency congestion, patient travel, working towards prevention and cure rather than treatment and sickness and overall healthcare costs by proving guiding solution. The main objective of this paper is to assist healthcare to anyone, anytime, and anywhere i.e. pervasive healthcare whileincreasing the coverage and the quality of healthcare by removing time, locational and other restraints. This includes prevention, healthcare maintenance and checkups, short-term monitoring, long term monitoring, emergency intervention, personalized healthcare monitoring, incidence detection and management, transportation and treatment with low cost, indigenous and guiding solution.

Figure 1 shows that the Blood Pressure Sensor input is detected and feeded to the Labview Software ${ }^{9}$, where the measured systolic and diastolic pressures value is obtained. Then the value is transferred wirelessly to the mobile phone where the guiding system mobile application needs to be executed for various solutions for controlling the blood pressure ${ }^{10}$.

\subsection{System Design for Blood Pressure Measurement}

Blood pressure is traditionally measured by sphygmomanometer consisting of an inflatable cuff to restrict blood flow, a mercury or mechanical nanometer to measure pressure, an inflation bulb to pump air, a valve for user to decrease the air pressure gradually and valves to connect the parts. Vernier Blood Pressure Sensor ${ }^{5}$ uses oscillometric method to measure the systemic arterial blood pressure in human in a non-invasively way which gives fairly accurate results but convenient and less painful measurement. SenSym SDX05D4 pressure transducer converts pressure measured in the cuff into an output voltage amplified to be linear to pressure ${ }^{11}$. In the oscillometric method, cuff pressure is increased rapidly to a much higher level than systolic arterial pressure to

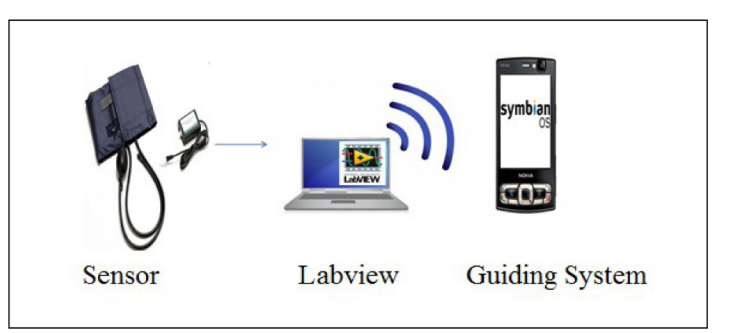

Figure 1. Overview diagram. 
stop blood flow. The cuff pressure slowly and constantly decreases that forces the blood flow in short pulses. As the cuff pressure continues to reduce, the pulses progressively achieve a maximum value and then drop until the occlusion is removed. These pressure pulses form an oscillating waveform describe systolic, diastolicpressures and MAP. The cuff pressure related to the maximum amplitude can estimate MAP. Using a known percentage of MAP, diastolic and systolic pressure is calculated. The cuff pressure waveform measured by sensor and transformed by interface can be shown using data collection software like LabVIEW. LabVIEW (Laboratory Virtual Instrumentation Engineering Workbench) developed by NationalInstrument is a platform and work environment for visual programming language ${ }^{12}$. LabVIEW programs are called virtual instruments (VIs) since their iconic look and function emulate the real instruments such as multimeters or oscilloscopes. Each VI has three components are block diagram, front panel, andconnector panel. The block diagram contains the code built in the user interface or front panel. Controls such as buttons, dials or knobs allow input of data into a running VI, and indicators are graphs, LEDs and others output display. The front panel can also serve as a programmatic interface. By connecting with hardware such as sensors, vision and motion control devices, LabVIEW can acquire, analyze, display and store data. In the blood pressure measurement, LabVIEW communicates with the blood pressure sensor through the SensorDAQ interface to collect the measured signals, calculate and display the experiment results.

\subsection{Guiding System}

The guiding system is the mobile application consists of the various precautions for the blood pressure measurement. The result obtained from the LabVIEW, is given to the guiding system through various wireless methods like Bluetooth/ Wi-Fi /GSM. The use case diagram in Figure 2 shows the role of the user in the Guiding system.

Once the user receives the BP value, it includes in the system and shows the range of that particular blood pressure the user has with various guiding solutions. By selecting the needed solution the result is shown as message alert for the user to do as precaution and treatment to control the blood pressure.User checks the range of the blood pressure obtained through the project flow diagram in Figure 3.

As the user opens the blood pressure measurement application in the mobile, both systolic and diastolic value

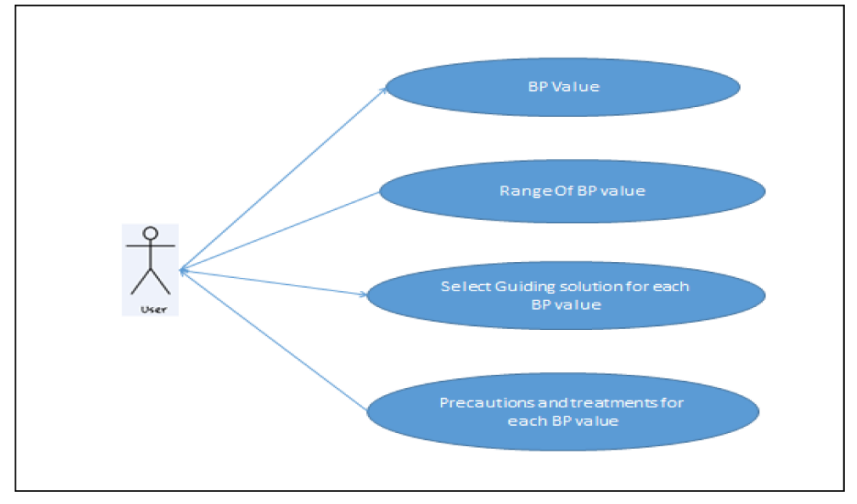

Figure 2. Use case Diagram.

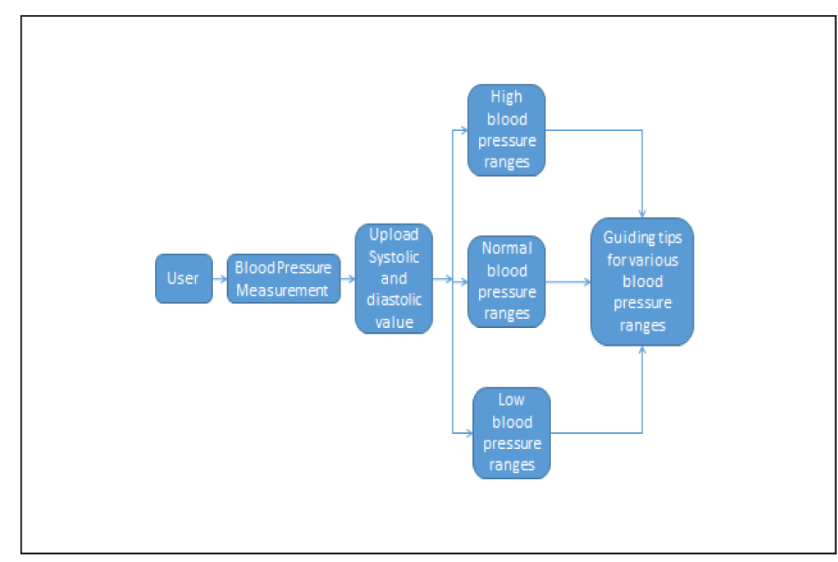

Figure 3. Guiding system project flow diagram.

is uploaded then its shows the various ranges of bloodpressure based on the chart shown in Figure 3, consist of high blood pressure ranges, Low blood pressure ranges and Normal blood pressure ranges are shown clearly with its effect ${ }^{13}$.

Based on the ranges of the blood pressure, the needed guiding solution is provided in the J2ME based mobile application using the Sun wireless tool kit ${ }^{14}$.The mobile application development tools of required Hardware and Software are shown in table 1 and 2

\section{Results}

The Graphical user interface based blood pressure measurement form consist of the Systolic and diastolic textbox for getting the value in the mobile application as shown in Figure 5

The next form of the guiding system by clicking submit option leads to the various guiding solution for the particular range of the blood pressure given as input is shown in Figure 6 


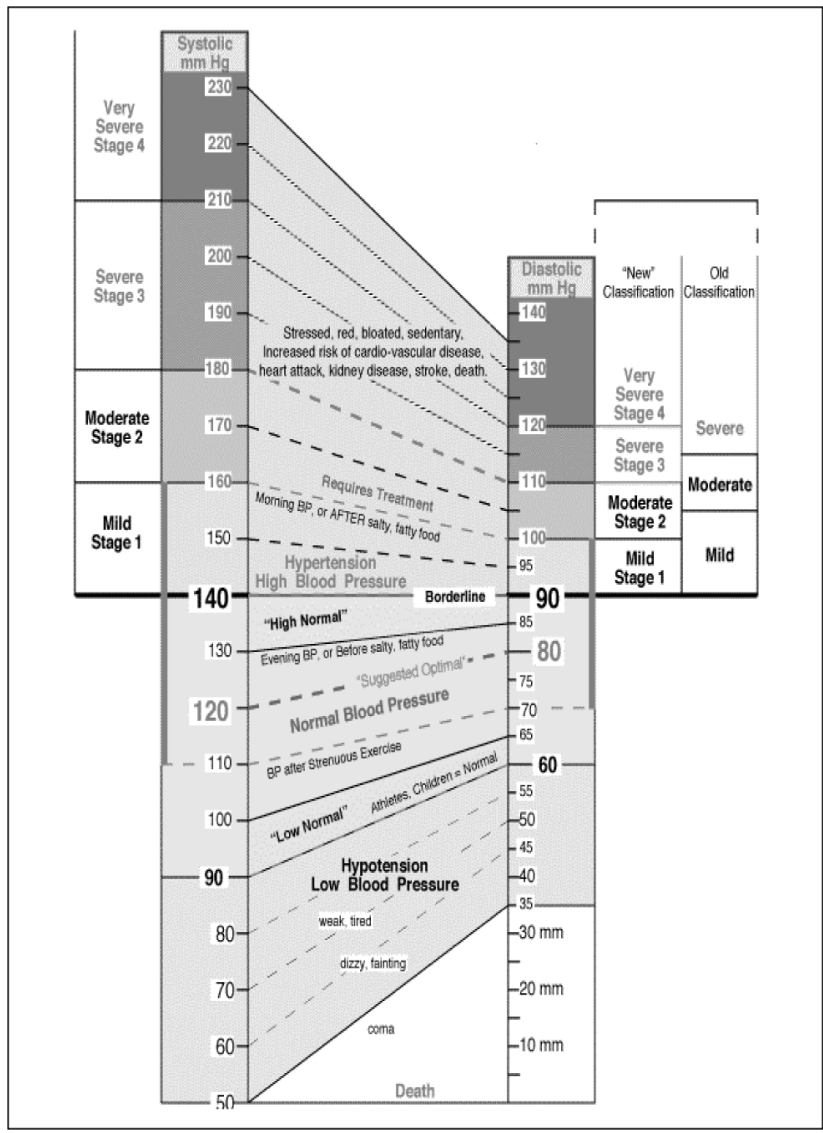

Figure 4. Blood pressure measurement chart.

Table 1. Hardware requirements

\begin{tabular}{lc}
\hline HARDWARE & REQUIREMENT \\
\hline $\begin{array}{l}\text { Processorspeed } \\
\text { RAMsize }\end{array}$ & $\begin{array}{c}\text { PentiumIIwithata speedof100MHzandabove. } \\
\text { 256MBandabove. }\end{array}$ \\
Display & 1024x768orhigherwith65536andmorecolours. \\
HardDisksize & 550Megabytesofmemoryandabove. \\
Handsetmodel & $\begin{array}{c}\text { Anymodelthatisjava-enabled(CLDC1.0and } \\
\text { MIDP1.0 orCLDC1.1.andMIDP2.0support), } \\
\text { withascreen resolutionof128x128orhigher. }\end{array}$ \\
\hline
\end{tabular}

Table 2. Software requirements

\begin{tabular}{ll}
\hline SOFTWARE & REQUIREMENT \\
\hline Operatingsystem & Microsoftwindows. \\
JavaDevelopmentKit & JDK1.5andabove. \\
WirelessToolkit & $\begin{array}{l}\text { SunJavaWirelessToolkit } \\
\text { forCLDC. }\end{array}$ \\
$\begin{array}{l}\text { IntegratedDevelopmentEnvir } \\
\text { onment(IDE) }\end{array}$ & Net beans \\
\hline
\end{tabular}

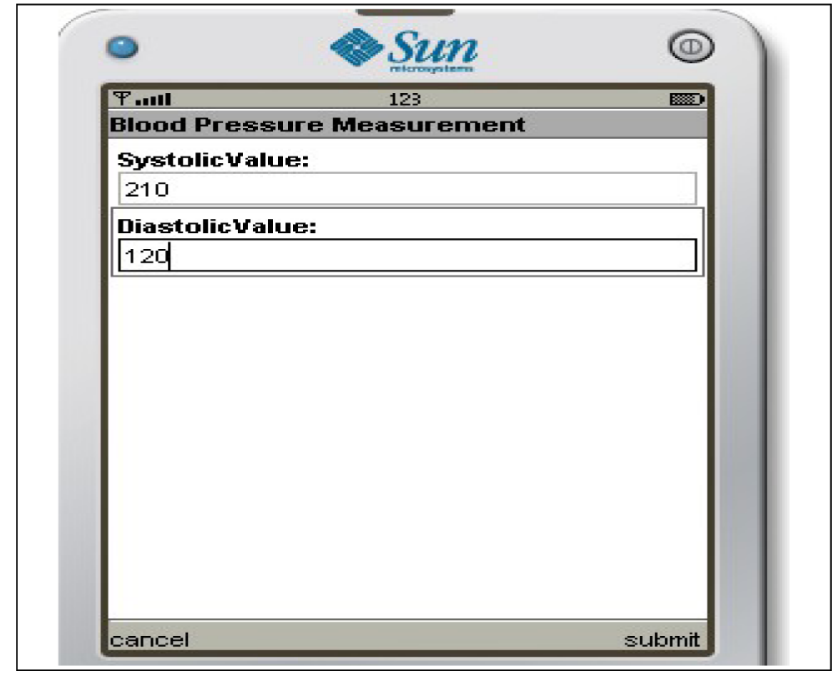

Figure 5. Snapshots of guiding system with blood pressure measurement form.

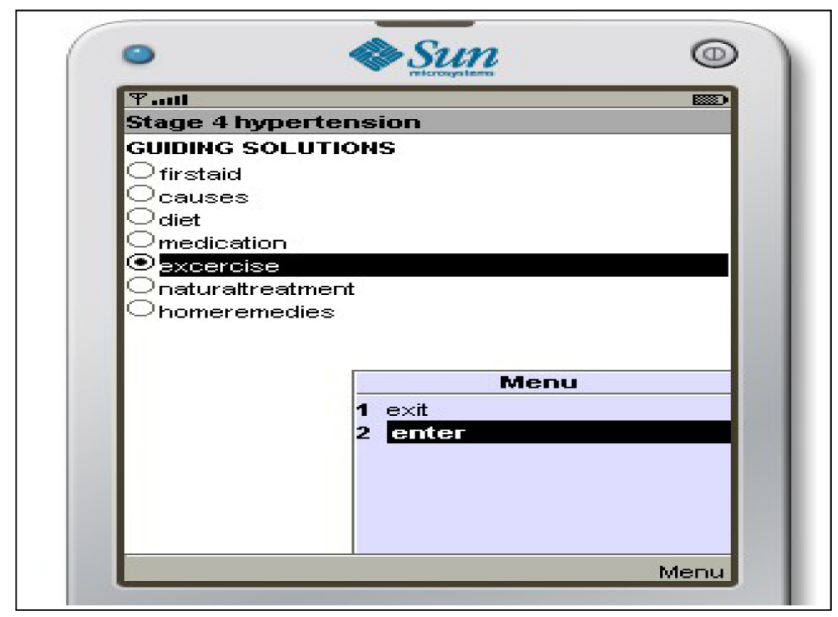

Figure 6. Snapshots of guiding system with various solutions to control blood pressure ranges.

On selecting the needed guiding solution of that particular range of blood pressure, the result is shown as the alert message (Figure 7) for controlling the blood pressure changes effectively ${ }^{15}$.

\section{Conclusion and Future work}

This paper presents the method of acquiring the information of the probability of blood pressure diseases using mobile guiding system with User Interface for blood pressure monitoring. The SGBPM MIDlet tested via simulation and its jar file can be used as application in mobile phones available. The future works can be extended to 


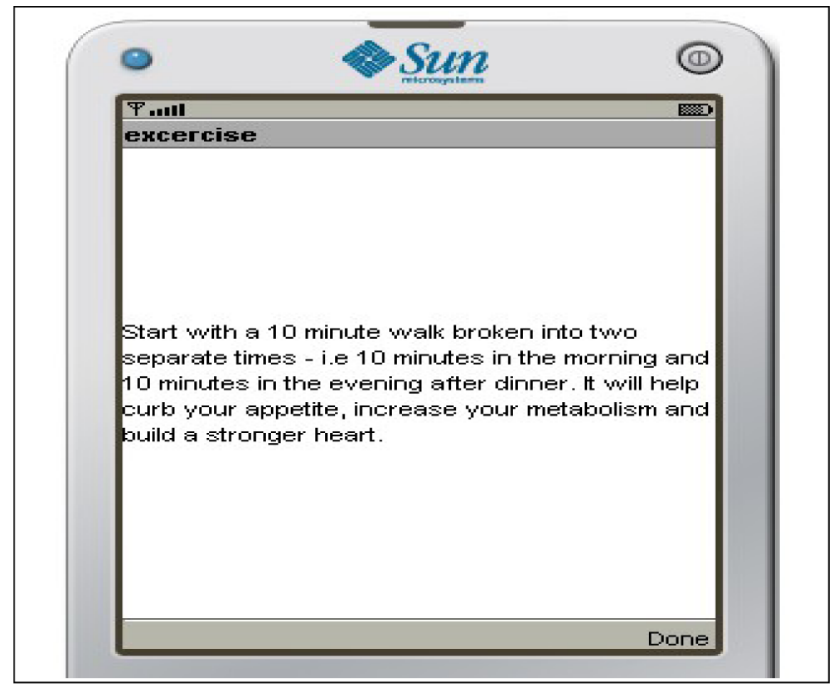

Figure 7. Snapshots of guiding system with alert message.

automatically detect the probability of blood pressure diseases using complete embedded system.

\section{References}

1. http://en.wikipedia.org/wiki/Remote_patient_monitoring

2. http://en.wikipedia.org/wiki/Remote_guidance

3. www.who.int media centre news releases 2012 world health.
4. Elina Mattila "Design and evaluation of a mobile phone diary for personal health management", Edita Prima Oy, Helsinki 2010.

5. http://www.vernier.com/products/sensors/bps-bta/

6. http://www.ni.com/labview/

7. James Keogh" J2ME: The Complete reference "McGrawHill, 2003.

8. Hun Shim, Hyo Min Kim, Sang Ha Song, Jung Hoon Lee, Joo HwanLee, Hyung Ro Yoon, Young Ro Yoon "Personalized HealthcareComment Service for Hypertension Patients Using Mobile Device"30th Annual International IEEE EMBS Conference Vancouver,British Columbia, Canada, 2008.

9. http://www.ni.com/data-acquisition/what-is/

10. http://www.java.com/en/download/faq/whatis_j2me.xml

11. http://www.technologyreview.in/article/405527/pervasivewireless/

12. Otuonye, A.I. "Improving University Education in Nigeria through Mobile Academic Directory".

13. http://www.vaughns-1-pagers.com/medicine/bloodpressure.htm

14. Seulki Lee, Jerald Yoo, and Hoi-Jun Yoo, "A Wearable Inductor Channel Design for Blood Pressure Monitoring System in Daily Life", 3rd International Conference on Pervasive Computing Technologies for Healthcare, Pervasive Health 2009, 2009; 1-4.

15. http://www.medicinenet.com/script/main/art. asp?articlekey=23356. 